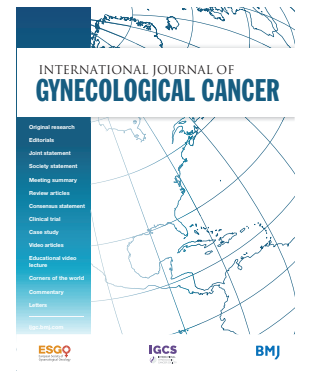

- Additional material is published online only. To view please visit the journal online (http://dx.doi.org/10.1136/ijgc2020-001384)

${ }^{1}$ Gynecologic Oncology Program, AdventHealth Cancer Institute, Orlando, Florida, USA ${ }^{2}$ Florida State University College of Medicine, Orlando, Florida, USA

${ }^{3}$ Augusta University Medical Center, Department of Obstetrics \& Gynecology, Augusta, Georgia, USA

\section{Correspondence to} Professor Sarfraz Ahmad, Gynecologic Oncology Program, AdventHealth Cancer Institute, Orlando, Florida FL 32804, USA sarfraz.ahmad@AdventHealth. com

Received 18 March 2020 Revised 9 June 2020 Accepted 11 June 2020 Published Online First 5 July 2020

Check for updates

(C) IGCS and ESGO 2020. № commercial re-use. See rights and permissions. Published by BMJ.

To cite: Paterniti TA, Ahmad S, Holloway RW. Int J Gynecol Cancer 2020;30:1189-1194.

\title{
Robotic-assisted laparoscopic splenectomy for recurrent ovarian cancer
}

\author{
Thomas A Paterniti, ${ }^{1,23}$ Sarfraz Ahmad (1) , ${ }^{1,2}$ Robert W Holloway ${ }^{1,2}$
}

\author{
Highlights \\ - Robotic splenectomy for recurrence of ovarian cancer was feasible in select patients. \\ - The technique of robotic splenectomy is briefly described with accompanying illustrations. \\ - Outcomes of robotic splenectomy for ovarian cancer recurrence are presented.
}

\section{ABSTRACT}

Purpose Recurrent ovarian cancer frequently involves the spleen. Our aims were to describe the technique of robotic-assisted laparoscopic splenectomy and to evaluate outcomes including progression-free and overall survival in patients who underwent this procedure for recurrent ovarian cancer.

Methods Chart reviews were performed on all consecutive patients who underwent robotic splenectomy (April 2012 to May 2019) for recurrent ovarian cancer. Patients had $\leq 3$ sites of disease and no ascites. Extent of disease was confirmed by positron emission tomographycomputed tomography (PET-CT) pre-operatively and platinum-doublet chemotherapy was initiated post-operatively. Peri- and post-operative outcomes, progression-free survival, and overall survival were assessed. Two video links are included to demonstrate variations in technique and anatomy.

Results A total of 10 patients were included. The median age was 63.5 years (range 46-74) and median body mass index was $30 \mathrm{~kg} / \mathrm{m}^{2}$ (range 21.5-40.1). Disease was limited to the spleen in seven patients and three had evidence of up to two other sites of disease on imaging. The median robotic splenectomy operative time was 159 min (range 112-214) that included laparoscopic lysis of adhesions prior to robotic port placement in seven cases, and excision of diaphragm or omental implants in three cases. There were no transfusions, laparotomy conversions, return to the operating room, abscesses, or pancreatic pseudocysts. The median length of stay was 2 days (range 1-4). The median time to resumption of chemotherapy was 40 days (range 25-78). After a median follow-up of 51 months (range 12-98), five patients had recurrence (two deaths, three alive with disease), with a median time to recurrence of 14 months (range 12-15). The median progression-free survival was 15 months (range 12-98) and the median overall survival was 51 months (range 12-98) post-splenectomy.

Conclusions Robotic splenectomy was feasible, achieving complete cytoreduction of splenic recurrent ovarian cancer, short hospital length-of-stay, and acceptable morbidity.

\section{INTRODUCTION}

The spleen is a frequent site of metastasis in nonhematologic cancers. Of all solid malignancies, splenic metastases are reported in $0.6-17 \%$ of patients in autopsy series. ${ }^{1-4} \mathrm{~A}$ Japanese study of upper abdominal screening ultrasounds performed on patients with a known cancer found an $11 \%$ incidence of splenic metastasis. ${ }^{5}$ Of all splenic metastases, $4-19 \%$ originated from an ovarian primary in autopsy studies ${ }^{134}$ compared with $27-43 \%$ in retrospective clinical series. ${ }^{67}$ This disparity likely reflects a selection bias within retrospective studies due to the surgical nature of ovarian cancer treatment. The incidence of ovarian cancer splenic metastasis varies by study type, occurring in $5-22 \%$ of autopsy studies ${ }^{238}$ compared with $3-7 \%$ in retrospective clinical series. ${ }^{7-9}$ In one autopsy study of 423 patients with ovarian cancer, there was no difference in the incidence of splenic metastases among different histologic subtypes of ovarian cancer. ${ }^{8}$

Although the benefit of primary cytoreduction in ovarian cancer is well-established, the role of secondary cytoreductive surgery for recurrent disease is controversial, especially given results from the phase III Gynecologic Oncology Group (GOG)-213 trial that reported no improvement in overall survival with secondary cytoreduction plus chemotherapy, compared with chemotherapy alone. ${ }^{9}$ Retrospective studies assessing secondary tumor debulking have been difficult to interpret and compare due to selection bias, heterogeneity of patients, and varying definitions of optimal cytoreduction, ranging from $<2 \mathrm{~cm}$ to no visible residual disease. ${ }^{10}$ Secondary cytoreductive surgery is most commonly performed for patients with isolated platinum-sensitive recurrent disease that is amendable to complete resection, and survival benefits have been reported in retrospective analyses. ${ }^{11}{ }^{12}$ However, the relatively recent use of bevacizumab as a component of chemotherapy regimens in ovarian cancer may have obscured the benefit of secondary cytoreduction in more recent studies. Patients who underwent secondary cytoreductive surgery followed by chemotherapy in the GOG-213 trial had no improvement in progression-free or overall survival compared with patients randomized to chemotherapy alone; however, $>80 \%$ of patients 


\section{Original research}

in both arms received bevacizumab during treatment and as maintenance. While there was also no improvement in overall survival for the subpopulation that underwent a complete gross resection (RO) compared with all patients randomized to chemotherapy, progression-free survival in the R0 surgical group did exceed that for patients receiving chemotherapy alone (hazard ratio (HR) 0.62 , $95 \% \mathrm{Cl} 0.48$ to 0.80 , median progression-free survival 22.4 vs 16.2 months, respectively),. ${ }^{9}$

There are limited reports of minimally invasive secondary cytoreduction splenectomy for gynecologic cancers. Eight cases of laparoscopic splenectomy in ovarian cancer have been described, ${ }^{13}$ and another video case report demonstrated the technical feasibility of robotic-assisted laparoscopic splenectomy in a patient with recurrent endometrial cancer. ${ }^{14}$ The primary aim of this investigation was to describe the technique of robotic-assisted laparoscopic splenectomy for recurrent ovarian cancer. Our secondary objectives were to analyze peri-operative clinical outcomes, progression-free survival, and overall survival for the first 10 patients who underwent this cytoreductive procedure.

\section{METHODS}

Retrospective chart reviews were conducted on the first 10 patients who underwent robotic-assisted laparoscopic splenectomy for a splenic recurrence of platinum-sensitive ovarian cancer at our hospital between April 2012 and May 2019. Patients for this study were identified in a contemporaneously managed robotic surgery database, initiated May 5, 2006. The Institutional Review Board approved the robotic surgery database and the retrospective analysis. Each patient had Eastern Cooperative Oncologic Group (ECOG) clinical performance status (0-1), platinum-sensitive highgrade epithelial ovarian cancers with a platinum-free interval $>12$ months, and one patient had recurrent granulosa cell tumor. All patients had three or fewer sites of intra-abdominal disease via positron emission tomography-computed tomography (PET-CT), no evidence of extra-abdominal metastases, and no evidence of ascites.

Robotic-assisted laparoscopic splenectomy was performed by a senior surgeon (RWH) with fellow assistance, and platinumdoublet chemotherapy was initiated post-operatively. Patient demographics, surgical, post-operative, and immediate follow-up data were collected from patient charts (electronic and paper) that were contemporaneously accessioned in a robotic surgery database. Peri-operative outcomes including estimated blood loss, transfusions, length-of-stay, conversion to laparotomy, and complications were abstracted from the charts. Disease-free survival was measured from the time of splenectomy until the clinical and radiographic diagnosis of recurrence. Overall survival was measured from the time of splenectomy until death. Follow-up was measured from the time of splenectomy until the most recent contact with the patient and/or family member. Time to recurrence was defined as the time from splenectomy to radiographic evidence of recurrence. Secondary chart reviews were conducted for analysis of treatment history, recurrence of disease, and survival. Data are presented as a percentage or as a median with a range for continuous variables, and as rates for discrete variables.

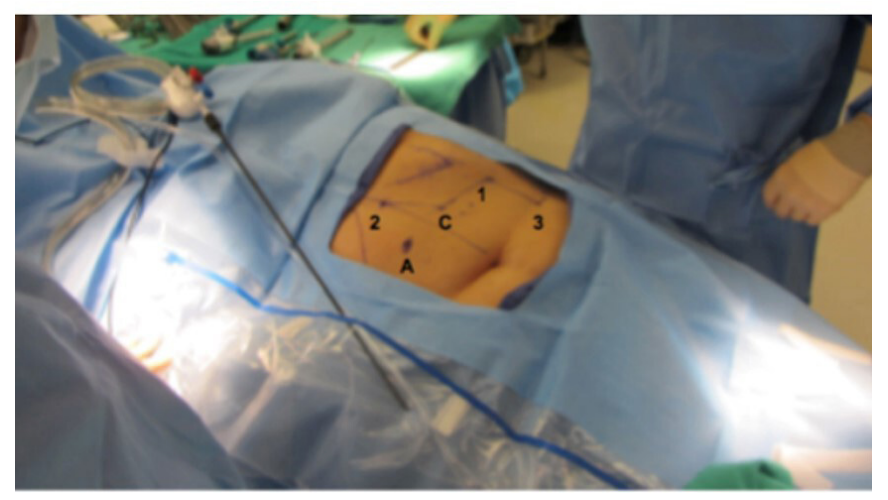

Figure 1 Selected image showing surgical positioning and trocar placement. Camera port is $15-18 \mathrm{~cm}$ from the left costal margin. The third arm is in the left flank. Accessory port is midway between camera and second arm in the right upper quadrant, used to retract stomach and provide suction.

\section{SURGICAL TECHNIQUE}

\section{Patient Preparation and Positioning}

Patients underwent mechanical bowel preparation with MiriLAX in clear liquid. Patients were placed in the dorsal supine lithotomy position in Allen stirrups with arms tucked and a kidney roll on the left flank. The operating table was jackknifed $15^{\circ}$ and rotated $30^{\circ}$ in right lateral tilt, with $15^{\circ}$ of reverse Trendelenburg in order to help expose the spleen. A vacuum bean-bag restraint was used in some cases, while other patients were padded on a standard table mattress, kidney roll, and secured with straps. Insufflation was performed with a $5 \mathrm{~mm}$ laparoscopic direct visualization port in the left upper quadrant near the mid costal margin. Washings were obtained. Laparoscopic adhesiolysis was performed when necessary for port access and the abdominal cavity was surveyed for absence of carcinomatosis.

Six cases were performed with the da Vinci Si unit docked over the patient's left shoulder, and the da Vinci Xi was used in four cases with the column side docked from the patient's right side, with the boom rotated to target the left upper quadrant. The camera port was placed approximately $15-18 \mathrm{~cm}$ from the mid-clavicular line at the left costal margin, near the midline, cephalad to the umbilicus with the other ports spaced 8-9cm apart as shown (Figure 1).

\section{Robotic Surgery Technique}

1. The lesser sac is exposed and entered with sharp dissection and cautery, separating any residual omentum from the transverse colon, and mobilizing the splenic flexure (Figure 2) caudally. In cases with prior complete omentectomy, the transverse colon mesentery is separated from the gastrocolic ligament in order to further mobilize the splenic flexure.

2. Next, the gastrosplenic ligament and the short gastric vessels within it are divided using either the robotic vessel sealer or the fenestrated bipolar grasper and monopolar shears. The gastrosplenic ligament is divided to the most cephalad portion of the gastric fundus near the diaphragm. The vessel sealer is the safer option for dividing short gastric vessels because the lateral heat spread is approximately $2 \mathrm{~mm}$.

3. Exposing the splenic hilum, splenic artery, and splenic veins is variable depending on the pathology. In general, it is safest to 


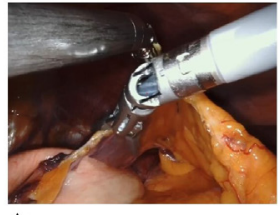

A.
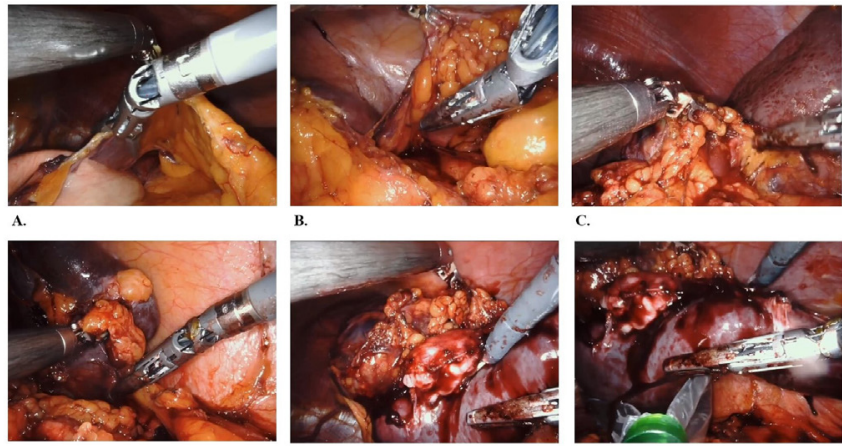

c.

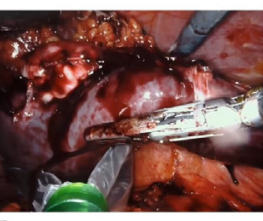

Figure 2 Selected images showing surgical technique.

(A) The lesser sac is exposed and entered, separating any residual omentum from the transverse colon. (B) The gastrosplenic ligament and the short gastric vessels within it are divided using either the robotic vessel sealer or bipolar cautery. (C) The splenic artery and vein are divided with a Hem-o-lock clip if possible; otherwise, they are divided with the robotic vessel sealer. (D) The posterior splenorenal ligament along with the inferior pole vessels are released. $(E)$ The spleen is seen here with the tumor mass in the splenic hilum. (F) The spleen is bagged and removed through a minilaparotomy incision.

expose the splenic artery from the ventral approach as it courses over the pancreas; but recognize that it will branch near the hilum in three to five separate smaller branches. The tapered end of the vessel sealer is ideal for dissecting and spreading soft tissue around the splenic artery and vein once the lesser sack of the peritoneum has been opened. While the vessel sealer is designed to seal vessels up to $7 \mathrm{~mm}$, it is prudent to clip the splenic artery prior to division with Hem-0-lok (Teleflex Medical, Morrisville, NC) when possible, as a measure of redundancy. First, the splenic artery is occluded and some time is given for the spleen to empty itself through the vein. This technique is demonstrated in online supplementary video case \#1.

4. After dividing the splenic artery and vein, the splenectomy is completed by elevating the splenic hilum, dividing posterior peritoneal attachments (splenorenal ligament), inferior pole vessels, and the splenophrenic attachments to the left diaphragm. The posterior approach allows better visualization of the most distal pancreas that is usually $1 \mathrm{~cm}$ from the hilum, and reduces the risk of pancreatic injury. In some cases, the hilum may be scarred from prior therapy with adjacent treated tumor, making identification of the individual arteries and veins difficult (online supplementary video case \#2). This case was accomplished using the vessel sealer without Hemolock clips, taking care to stay close to the hilum in order to avoid injury to the pancreas. If the distal pancreas requires resection, a vascular endo-stapler should be used.

5. Hemostasis is achieved on the diaphragm with bipolar and monopolar cautery. The spleen is then placed in a $15 \mathrm{~cm}$ Endobag, elevated to the abdominal wall, and removed through a 10$14 \mathrm{~cm}$ mini-laparotomy incision. We prefer using a midline port and incision for ease of closure. No surgical drains were placed in this series.
Table 1 Patient and tumor characteristics

\begin{tabular}{|c|c|}
\hline Characteristics & $\begin{array}{l}\text { Median (range), } \\
\text { n (\%) }\end{array}$ \\
\hline All cases, $n$ & 10 \\
\hline Age, year & $63.5(46-74)$ \\
\hline BMI $\left(\mathrm{kg} / \mathrm{m}^{2}\right)$ & $30.0(21.5-40.1)$ \\
\hline Platinum-free interval (months) & $24(17-145)$ \\
\hline Pre-operative CA125 (U/mL) & $43(7-1292)$ \\
\hline \multicolumn{2}{|l|}{ Cytoreduction status } \\
\hline Secondary & $8(80 \%)$ \\
\hline Tertiary & $2(20 \%)$ \\
\hline \multicolumn{2}{|l|}{ FIGO stage at diagnosis } \\
\hline IIC & $1(10 \%)$ \\
\hline IIIB & $2(20 \%)$ \\
\hline IIIC & $6(60 \%)$ \\
\hline IV & $1(10 \%)$ \\
\hline \multicolumn{2}{|l|}{ Histopathologic subtype } \\
\hline Serous & $8(80 \%)$ \\
\hline Granulosa cell & $1(10 \%)$ \\
\hline $\begin{array}{l}\text { Malignant mixed Müllerian tumor } \\
\text { (MMMT) }\end{array}$ & $1(10 \%)$ \\
\hline \multicolumn{2}{|l|}{ Tumor grade } \\
\hline 1 & $1 / 10(10 \%)$ \\
\hline 2 or 3 & $9 / 10(90 \%)$ \\
\hline \multicolumn{2}{|l|}{ Status of primary debulking } \\
\hline Ro & $9(90 \%)$ \\
\hline $\mathrm{R} 1-2$ & $1(10 \%)$ \\
\hline \multicolumn{2}{|l|}{ Status post-splenectomy } \\
\hline $\mathrm{RO}$ & $10(100 \%)$ \\
\hline \multicolumn{2}{|l|}{ Recurrence post-splenectomy } \\
\hline Yes & $5 / 10(50 \%)$ \\
\hline No & $5 / 10(50 \%)$ \\
\hline
\end{tabular}

BMI, body mass index; CA125, cancer antigen 125; FIGO, International Federation of Gynecology and Obstetrics; R0, resection for cure or complete remission; $\mathrm{R} 1$, resection to microscopic residual tumor; R2, resection to macroscopic residual tumor.

\section{RESULTS}

Ten consecutive patients with recurrent ovarian cancer involving the spleen and $\leq 3$ sites of disease on PET-CT underwent roboticassisted laparoscopic splenectomy. Eight splenectomies were performed as a secondary cytoreduction and two as a tertiary cytoreduction. Pre-operative characteristics including the original staging and pathology characteristics are summarized in Table 1. The median age was 64 years (range 46-74) and the median body mass index was $30.0 \mathrm{~kg} / \mathrm{m}^{2}$ (range 21.5-40.1). The median platinum-free interval was 25 months (range 17-145) and all patients were platinum-sensitive. One of the patients had International Federation of Gynecologic Oncology (FIGO) stage II disease at the original diagnosis, eight were stage III, and one was stage IV. 
Table 2 Surgical outcomes of robotic-assisted

laparoscopic splenectomy

\begin{tabular}{ll}
\hline Characteristics & Median (range) \\
\hline Splenectomy operative time (min) & $159(112-214)$ \\
Estimated blood loss $(\mathrm{mL})$ & $100(0-600)$ \\
$\begin{array}{l}\text { Post-operative decrease in hemoglobin }(\mathrm{g} / \\
\mathrm{dL})\end{array}$ & $1.0(-0.1$ to 2.3$)$ \\
Tumor size, cm (greatest diameter) & $3.1(1.5-6.5)$ \\
Hospital length-of-stay (days) & $2(1-4)$ \\
\hline
\end{tabular}

*Three cases involved separate procedures performed by other surgeons (robotic cholecystectomy, combined robotic and open ventral herniorrhaphy, and robotic sigmoid colectomy with anastomosis). Splenectomy times included port placements and closures, lysis of adhesions, and associated oncologic procedures described in the text.

Eight tumors had serous histology, one patient had granulosa cell tumor, and one had a malignant mixed Müllerian tumor. The median pre-operative cancer antigen 125 (CA125) was $43 \mathrm{U} / \mathrm{mL}$ (range 7-1292) and the patient with the granulosa cell tumor had a preoperative serum inhibin of $193 \mathrm{pg} / \mathrm{mL}$. Radiographically, disease was limited to the spleen in seven patients, while three had PET-CT evidence of extra-splenic metastasis, including one implant along the left hemi-diaphragm, one metastasis to a portal triad lymph node, and one sigmoid colon mesenteric mass.

Laparoscopic lysis of adhesions was performed in seven of the 10 cases in order to improve exposure for robotic port placement. Two patients had intraperitoneal port-catheter systems removed, and one underwent intraperitoneal port placement for chemotherapy following splenectomy. Three patients underwent additional procedures at the time of splenectomy by general surgeons that included a robotic sigmoid colon resection and anastomosis, a robotic cholecystectomy, and a component-separating ventral hernia repair with mesh performed robotically and by laparotomy. A complete tumor resection (R0) was achieved in all patients.

Surgical peri-operative outcomes are summarized in Table 2. For the three patients undergoing additional general surgery procedures, the approximate time spent during the splenectomy was determined using anesthesia records. The median estimated operative time of splenectomy including lysis of adhesions for the 10 cases was 159 min (range 112-214), the median estimated blood loss was $100 \mathrm{~mL}$ (range 0-600), and the median tumor size was $3.1 \mathrm{~cm}$ (range 1.5-6.5). No cases were converted to laparotomy for the performance of the splenectomy; however, one case was converted to laparotomy in order to complete a ventral hernia repair following the splenectomy.

One patient developed a symptomatic pleural effusion that required thoracentesis on post-operative day 3 . The median decrease in hemoglobin on post-operative day 1 was $1.0 \mathrm{~g} / \mathrm{dL}$ (range -1 to 2.3). There were no transfusions, return to the operating room, abscesses, or pancreatic pseudocysts. The median hospital length-of-stay was 2 days (range 1-4), and all patients were cleared for chemotherapy by day 21 post-operative visits. The median time to resumption of chemotherapy was 40 days (range 25-78). Two patients were delayed for placement of venous access ports, two electively delayed to complete planned travel prior to
Table 3 Clinical outcomes of robotic-assisted laparoscopic splenectomy

\begin{tabular}{ll}
\hline Characteristic & $\begin{array}{l}\text { Median } \\
\text { (range) }\end{array}$ \\
\hline Disease-free survival (months), $\mathrm{n}=10$ & $15(6-92)$ \\
Time to recurrence (months), $\mathrm{n}=5$ & $14(12-15)$ \\
Overall survival (months), $\mathrm{n}=10$ & $47(6-92)$ \\
Follow-up in all patients (months) & $47(6-92)$ \\
Follow-up in disease-free patients (months) & $22(6-55)$ \\
$\mathrm{n}=5$ & \\
\hline
\end{tabular}

initiation of therapy, and three were delayed establishing care with local oncologists. All patients received platinum-doublet chemotherapy following cytoreductive surgery, and only one patient did not complete the planned six cycles of therapy, choosing instead to change to alternative therapies after the fourth cycle.

Clinical outcomes are summarized in Table 3. The median progression survival was 15 months (range 12-98) and the median overall survival was 51 months (range 12-98). After a median follow-up of 51 months (range 12-98), five patients remained disease-free. The median follow-up in those who remained disease-free was 45 months (range 12-98). The median time-to-recurrence was 14 months (range 12-15), with two patients deceased and three alive on additional therapy.

\section{DISCUSSION}

The use of minimally invasive surgery in the treatment of gynecologic malignancies has steadily increased over the past two decades, with multiple studies demonstrating feasibility for select patients with ovarian cancer. ${ }^{15-18}$ Robotic-assisted laparoscopy offers well-known advantages of smaller incisions with decreased post-operative pain, as well as decreased blood loss and lengthof-stay compared with laparotomy, often resulting in swifter resumption of adjuvant chemotherapy. The median operative time in our series was longer than the $75 \mathrm{~min}$ (range 25-360) reported by Corcione et $\mathrm{al}^{19}$ in a retrospective review of 300 laparoscopic splenectomies performed for hematologic disorders. However, our operative times were comparable to the 200 min (range 80-275) for laparoscopic splenectomy in recurrent ovarian cancer reported by Gallotta et al. ${ }^{13}$ The estimated blood loss and hospital length of stay in our series were also very similar to that seen in laparoscopic splenectomy. ${ }^{13}$

Controversy remains as to which patients are ideal candidates for minimally invasive surgery in recurrent ovarian cancer. There are no prospective trials that address minimally invasive secondary cytoreduction. A retrospective cohort study found both laparoscopy and robotic surgery preferable to laparotomy with less blood loss and shorter hospital stay, provided patients had minimal sites of disease. ${ }^{17}$ The optimal mode of pre-operative imaging prior to secondary cytoreduction is also debated. CT scans are considered standard of care in the pre-operative evaluation of patients; however, they are limited in the detection of $<1 \mathrm{~cm}$ peritoneal, bowel serosa, and mesentery metastases. Both PET-CT and magnetic resonance imaging have greater sensitivity for detection 
of carcinomatosis than $\mathrm{CT}^{20-22}$ PET-CT can detect metastases in normal appearing lymph nodes $<1 \mathrm{~cm},{ }^{22}$ and also has the advantage of detecting distant metastases that could obviate the need for secondary cytoreduction.

The overarching question is whether secondary cytoreduction can provide a survival advantage over chemotherapy alone for patients with platinum-sensitive disease. DESKTOP II established a prospectively validated tool for predicting complete surgical resection during secondary cytoreductive surgery based on three criteria: good ECOG performance status (0), macroscopically complete primary cytoreduction at the initial debulking, and the absence of ascites $>500 \mathrm{~mL}^{23}$ In addition to these criteria, we limited surgery to patients with no more than three sites of disease and platinumfree intervals $>12$ months. Patients who were able to undergo a complete resection in GOG-213 had improved progression-free survival compared with patients randomized to chemotherapy alone; however, this did not result in better overall survival.

Some may question the advisability of performing splenectomy for secondary cytoreduction following the publication of GOG-213 results. A complete critique of GOG-213 is beyond the scope of this paper; however, the criteria for determining a patient's candidacy for cytoreduction were not well defined, and the study took over 10 years to complete, possibly introducing variability in patterns of care. R0 resections were accomplished in only $67 \%$ of cases. In addition, $84 \%$ of patients received bevacizumab, which may have overshadowed any benefits of cytoreduction previously recognized in retrospective studies. In contrast, the DESKTOP III trial reported improved progression-free survival and overall survival for patients randomized to secondary cytoreduction compared with chemotherapy alone (HR $0.76, p=0.03$, and $H R 0.66, p<0.001$, respectively) (Du Bois et al, American Society of Clinical Oncology (ASC0) 2020 Annual Meeting, Oral Abstract \# 6000). Importantly, patients who had residual disease after surgery had worse overall survival than patients with chemotherapy alone, or complete resection (median overall survival 28.8, 46.2, and 60.7 months, respectively). Therefore, patient selection for resectability and avoidance of complications are key to achieving enhanced survival. Patients in our study had disease limited to the spleen in the majority of cases, and the remaining had three or fewer lesions that were deemed resectable. Minimally invasive robotic splenectomy was accomplished with low morbidity and a short length-of-stay, factors that should be considered with respect to an analysis of therapeutic benefit. Chemotherapy delay may negatively impact survival and attempts to initiate chemotherapy within 28 days should be discussed with patients, as several of our patients delayed unnecessarily.

In a retrospective analysis of platinum-resistant patients with isolated disease, secondary cytoreduction was also associated with improved progression-free and overall survival compared with similar patients who were managed with chemotherapy alone..$^{24}$ It is possible that some patients with less platinum-sensitive epithelial ovarian cancer (6-12 months platinum-free interval) could benefit from complete secondary cytoreduction, because the expected improvements in time to progression from chemotherapy would be less. Other patients with less common histologies such as granulosa cell tumors, low-grade serous cancers, and mucinous carcinomas that are less sensitive to chemotherapy might also benefit from secondary cytoreduction. Nevertheless, if secondary cytoreduction is contemplated, cases should be selected with isolated or limited disease as described in DESKTOP $\|,{ }^{23}$ with the anticipation of complete surgical resection in order to achieve the most benefit.

\section{CONCLUSIONS}

Our study with only 10 cases represents the largest case series of robotic-assisted laparoscopic surgery for splenic recurrence of ovarian cancer, emphasizing the highly selected population who underwent this procedure. Robotic-assisted laparoscopic splenectomy was feasible, achieving complete cytoreduction of recurrent ovarian cancer, with short hospital length-of-stay and acceptable morbidity.

Contributors TAP and RWH designed the study, and subsequently all the authors participated in the data collection, analyses, discussion on results and interpretation, and help drafting the manuscript.

Funding The authors have not declared a specific grant for this research from any funding agency in the public, commercial or not-for-profit sectors.

Competing interests $\mathrm{RWH}$ received compensation for advanced training programs from Intuitive Surgical, Inc (Sunnyvale, CA) and speaking honoraria from Bard-Davol (Warwick, Rl) during the time these surgeries occurred.

Patient consent for publication Not required.

Provenance and peer review Not commissioned; externally peer reviewed.

Data availability statement Data are available upon reasonable request.

\section{ORCID iD}

Sarfraz Ahmad http://orcid.org/0000-0002-5180-0409

\section{REFERENCES}

1 Abrams HL, Spiro R, Goldstein N. Metastases in carcinoma; analysis of 1000 autopsied cases. Cancer 1950;3:74-85.

2 Falk S, Biegler T, Stutte HJ. [Tumor metastases in the spleen] Pathologe 1987;8:85-90.

3 Hirst AE, Bullock WK. Metastatic carcinoma of the spleen. Am J Med Sci 1952;223:414-7.

4 Lam KY, Tang V. Metastatic tumors to the spleen: a 25-year clinicopathologic study. Arch Pathol Lab Med 2000;124:526-30.

5 Ishida H, Konno K, Ishida J, et al. Isolated splenic metastases. J Ultrasound Med 1997;16:743-9.

6 Gatenby PAC, Mudan SS, Wotherspoon AC. Splenectomy for nonhaematological metastatic malignant disease. Langenbecks Arch Surg 2011;396:625-38.

7 Morris M, Gershenson DM, Burke TW, et al. Splenectomy in gynecologic oncology: indications, complications, and technique. Gynecol Oncol 1991;43:118-22.

8 Rose PG, Piver MS, Tsukada Y, et al. Metastatic patterns in histologic variants of ovarian cancer. An autopsy study. Cancer 1989;64:1508-13.

9 Coleman RL, Spirtos NM, Enserro D, et al. Secondary surgical cytoreduction for recurrent ovarian cancer. $N$ Engl J Med 2019;381:1929-39.

10 Munkarah AR, Coleman RL. Critical evaluation of secondary cytoreduction in recurrent ovarian cancer. Gynecol Oncol 2004;95:273-80.

11 Al Rawahi T, Lopes AD, Bristow RE, et al. Surgical cytoreduction for recurrent epithelial ovarian cancer. Cochrane Database Syst Rev 2013;194.

12 Bristow RE, Puri I, Chi DS. Cytoreductive surgery for recurrent ovarian cancer: a meta-analysis. Gynecol Oncol 2009;112:265-74.

13 Gallotta V, Nero C, Lodoli C, et al. Laparoscopic splenectomy for secondary cytoreduction in ovarian cancer patients with localized spleen recurrence: feasibility and technique. J Minim Invasive Gynecol 2016;23:425-8.

14 Gallotta V, D'Indinosante M, Nero C, et al. Robotic splenectomy for isolated splenic recurrence of endometrial adenocarcinoma. J Minim Invasive Gynecol 2018;25:774-5.

15 Amara DP, Nezhat C, Teng NN, et al. Operative laparoscopy in the management of ovarian cancer. Surg Laparosc Endosc 1996;6:38-45. 


\section{Original research}

16 Escobar PF, Levinson KL, Magrina J, et al. Feasibility and perioperative outcomes of robotic-assisted surgery in the management of recurrent ovarian cancer: a multi-institutional study. Gynecol Oncol 2014;134:253-6.

17 Magrina JF, Cetta RL, Chang Y-H, et al. Analysis of secondary cytoreduction for recurrent ovarian cancer by robotics, laparoscopy and laparotomy. Gynecol Oncol 2013;129:336-40.

18 Holloway RW, Brudie LA, Rakowski JA, et al. Robotic-assisted resection of liver and diaphragm recurrent ovarian carcinoma: description of technique. Gynecol Oncol 2011;120:419-22.

19 Corcione F, Pirozzi F, Aragiusto G, et al. Laparoscopic splenectomy: experience of a single center in a series of 300 cases. Surg Endosc 2012;26:2870-6.

20 Rizzo S, Del Grande M, Manganaro L, et al. Imaging before cytoreductive surgery in advanced ovarian cancer patients. Int $J$ Gynecol Cancer 2020;30:133-8.
21 van 't Sant I, Engbersen MP, Bhairosing PA, et al. Diagnostic performance of imaging for the detection of peritoneal metastases: a meta-analysis. Eur Radiol 2020;30:3101-3112.

22 Sanli Y, Turkmen C, Bakir B, et al. Diagnostic value of PET/CT is similar to that of conventional MRI and even better for detecting small peritoneal implants in patients with recurrent ovarian cancer. Nucl Med Commun 2012;33:509-15.

23 Harter P, Sehouli J, Reuss A, et al. Prospective validation study of a predictive score for operability of recurrent ovarian cancer: the Multicenter Intergroup Study DESKTOP II. A project of the AGO Kommission OVAR, AGO Study Group, NOGGO, AGO-Austria, and MITO. Int J Gynecol Cancer 2011;21:289-95.

24 Petrillo M, Pedone Anchora L, Tortorella L, et al. Secondary cytoreductive surgery in patients with isolated platinum-resistant recurrent ovarian cancer: a retrospective analysis. Gynecol Oncol 2014;134:257-61. 